\title{
SasyaSneha - An Approach for Plant Leaf Disease Detection
}

\author{
Dr. Paul P. Mathai ${ }^{1}$, Aiswarya S. ${ }^{2}$, Aiswarya J.G. ${ }^{3}$, Athira Saiju ${ }^{4}$, Anagha Clare Antony ${ }^{5}$ \\ ${ }^{1}$ Federal Institute of Science and Technology, India, paul.mathai22@gmail.com \\ ${ }^{2}$ Federal Institute of Science and Technology, India, aiswaryasethu1997@gmail.com \\ ${ }^{3}$ Federal Institute of Science and Technology, India, aiswarya.jgopi@gmail.com \\ ${ }^{4}$ Federal Institute of Science and Technology, India, athirasaiju97@gmail.com \\ ${ }^{5}$ Federal Institute of Science and Technology, India, anaghaclareantony@gmail.com
}

\begin{abstract}
The productivity in agricultural field is the one on which economy is highly determined. It may be the reason why disease identification in plants plays a good role in the field of agriculture. If we are not giving proper care, then it creates serious problems on plants and due to this the product quality, quantity or productivity will be affected. In a large portion of the fields, real gains have been acquired by the properties of computational intensity of Deep Learning systems for settling master errands. Deep Learning technology is used in the area of agriculture to solve the problem of diagnosing the plant disease based on the image taken in Smartphone. The images of leaves of healthy and diseased plants were used for plant disease detection and recognition by making use of convolutional neural network models as the learning tool. The total preparing of the models was finished with the utilization of a dataset of 1000 pictures, containing 4 distinct plants including healthy plants. The proposed system is expected to give a significant high success rate. This can be used as an advisory or early warning tool, and this approach could be further expanded to support an advanced plant disease identification system to operate in real cultivation conditions.
\end{abstract}

Key words: Deep learning, Convolutional neural networks, Phenotyping, SVM classifier.

\section{INTRODUCTION}

Modern technologies have given human society the skill to make enough food to meet the demand of more than 7 billion people. About $70 \%$ of the population depends on agriculture since India is a cultivated country. Plant diseases lead to the remarkable reduction in both the quality and quantity of agricultural products. Food security stays compromised by various elements including environmental change, the decrease in pollinators, plant infections, and others. Plant illnesses are not just a danger to food security at the worldwide scale, yet can likewise have tragic results to poor farmers whose presence rely upon solid yields. The indicative procedure for example acknowledgment of indications and signs is intrinsically visual and requires natural judgment just as the utilization of logical strategies. The studies of visually observable patterns [1] on the plant leaves were referred on the studies of plant disease. Successful cultivation of crops in the farm can be ensured by monitoring of disease on plants. In early days, the observing and examination of plant diseases [2] were done physically by the mastery individual in that field. This requires huge measure of work and furthermore requires intemperate handling time.

Disease identification [3] and providing required nutrients to them become an important part of farming. When the diagnosis is done using naked eye and therefore there are high chances of wrong diagnosis. Verbal information is usually not scientific and not accurate leading to treating a wrong disease with wrong chemicals and eventually harming the environment. Depending on naked eye perception [4] by specialists to recognize plant ailments can be restrictively costly particularly in creating nations. The plant infection recognition utilizes the picture preparing strategies. The key to prevent the agriculture loss is the detection and classification of leaf diseases accurately. A mapping between sources of information, for example, a picture of a disease influenced plant to a yield, for example, a harvest illness pair is given by neural systems [5]. The hubs in a neural system are scientific capacities that take numerical contributions from the approaching edges, and give a numerical yield as an active edge. Profound neural systems are essentially mapping the information layer to the yield layer over a progression of stacked layers of hubs. The test is to make a profound system so that both the structure of the system just as the capacities (hubs) and edge loads accurately map the contribution to the yield. Profound neural systems were prepared by tuning the system parameters in a manner with the goal that the mapping improves during the preparation procedure. The combination of increased mobile penetration and internet use together with recent advances in computer vision through 
deep learning has made opportunities to exist for Smartphone based disease detection and phenotyping.

\section{LITERATURE REVIEW}

There is a novel picture handling calculation dependent on applicant hotspot recognition in mix with factual surmising strategies to handle disease identification in wild conditions. Another technique include the use of convolutional neural network models to perform detection of several plant diseases and identify the nature of an illness by examination of the symptoms using several images of leaves, which should include both its healthy and diseased textures, where diagnosis done through deep learning methodologies. Preparing of the models was performed with the utilization of an open dataset of pictures, containing 25 unique plants in a lot of 58 particular classes of [plant, disease] mixes, including sound plants.

Another approach is where the captured image of affected leaf is first preprocessed with an image processing algorithm enhance with Gaussian smoothing operator is a 2-D sophistication operator for smoothened images and remove noise. Subsequent to auditing all previously mentioned systems and techniques we can infer that there are a few different ways by which we can perform location of maladies that happen in plants. Every ha a few points of interest just as confinements. In this way, there is extent of progress in the current research. Picture preparing is a method which improves all current research and which gives quick and precise consequence of plant maladies. Subsequent to assessing all previously mentioned strategies and techniques, we can reason that there are a few different ways by which discovery of sickness of plants should be possible. Every ha a few preferences just as impediments. Picture preparing is a procedure which improves all exploration which has target reality and gives quick and precise aftereffect of plant maladies. Deep learning technique was found to be most accurate in predicting the disease from the captured image of a leaf.

Various designers have experienced automated plant disease distinguishing proof by mechanized visual analytic strategies. One among them was an android application called Plantix. It is a portable harvest warning for farmers and nursery workers which analyze plant diseases, bug harmed and insufficiencies influencing yields and offers relating treatment measures. It uses neural networks for training the model. We must capture the focused and clear image of the plant leaf to be diagnosed or could be imported from gallery. The application then asks for matches with their images from dataset. Hence, farmer has to manually identify the disease concerned with the leaf by checking matches.

Johannes et al. describes a technique for the identification of early symptoms of plant diseases. A variety of computerized visual diagnostic methods were evolved from different researches to address automated plant disease detection. One among them was employing Mobile capture devices to diagnose plant diseases. In this technique, an image processing calculation dependent on candidate hotspot recognition [6] as a team with some measurable derivation strategies were utilized to handle disease recognition in wild conditions. Estimating of plant disease is essentially isolated into three sections, to be specific, estimating of the occurrence, seriousness and yield loss. Despite the fact that it is basic to distinguish plant diseases in a beginning period to avoid yield loss, it is practically inconceivable and requires learning since the manifestations are not yet grown completely. Thus there is a need to give plant infection location instrument dependent on image handling. [7]

It gives an understanding into a trainable framework to distinguish plant sicknesses which has been approved in three European endemic wheat diseases by picture handling based strategies in blend with statistical inference methods. [8] Plant infection recognizable proof, as utilized here, incorporates the assurance of a probability that a specific ailment is available. Despite the fact that other sensor gadgets for immediate or aberrant shading variety recognition could give valuable data as seriousness and spreading in the plant or yield, they don't create enough data to analyze a particular harm in the plant, including biotic (disease, pest and weeds) and abiotic risks. Consequently, there is a need to give picture handling based plant sickness recognizable proof, to analyze diseases in their initial advancement stages to have the option to respond in time with harvest assurance applications.

Ferentinos et al. describes about the automated plant disease detection system which is implemented using Deep learning model which includes convolutional neural network as a learning tool [9]. CNN is trained with simple images of leaves which contain both healthy and diseased which was taken in laboratory and field conditions[10].This model was developed as an advance version of model designed by Mohanty, which compared only two architectures of CNN with 26 plant diseases and database with images of 14 plants, but the problem was that even though it gave 99.35 percentage of success rate, it was not able to use on real time conditions as the experiment was conducted only on laboratory images. Here database includes around 87,848 photographs which include both of healthy and infected plants in both conditions were totally it contains 25 plant species and this database is used for training and testing. The database is again categorized as 58 classes where each class includes a pair definition of plant and disease and also some classes include healthy plants. This database is again then divided into two parts: training set and testing set (divided as per 80/20 ratio). Thus, two CNN models were developed: one which was trained on laboratory images and tested with field ones and the other which was trained on field or real time cultivation images and tested on laboratory images. 
Nti et al. describes about an idea to identify diseases on crops and offer extra classification of malady. Here, the image taken of affected leaf will be first preprocessed with an image processing algorithm where it can be enhanced with Gaussian smoothing operator is a 2-D sophistication operator for smoothened images and remove detail (higher frequencies) and noise. [11] A radiation source helps to lit up the visual portrait of an image, and hence forms the image and all image formation has the following essentials, an object, a particle emission source and an image creation scheme.

The system is defined to give the disease and severity. It contains a Classification framework that prepares a 5 class order framework to choose the status of disease of a plant. The 5 classes introduce a wellbeing class and 4 illness class. It is likewise used to arrange distinctive seriousness levels for any of the 4 infections. Seriousness levels are assigned classes $1-5,1$ being a solid plant, 5 being a seriously infected plant. Due to extreme drought cassava crop was threatened with diseases particularly Cassava mosaic disease (CMD), Cassava brown streak disease (CBSD), Cassava bacterial blight (CGM) and Cassava green mite (CBB). Godliver, Owomugisha and Ernest Mwebaze focused on the most divesting diseases among the 4.Their paper mainly used the machine learning techniques to find and group the healthy and diseased plants leaf. Automatic recognition of diseases and its severity are the two outputs of their system.

\section{PROPOSED METHOD}

The Design and implementation can be primarily divided into 3 parts: Image capture phase, disease detection phase and remedy recommendation phase. Image Capture phase captures image using mobile application or allows user (mainly farmers) to choose an already taken image from gallery. This image after preprocessing is fed into the second part of our design that is diseased detection phase (SVM classifier), where SVM has been learned with images and their classes in the learning phase. From this learned knowledge, classifier classifies and predict class label for the new unseen data (image from first part). According to the class label predicted, looks whether it is healthy or diseased and provide corresponding remedy for the diseased leaf, specifying the disease and remedy to cure it. The user, usually farmers, captures the focused image of a leaf which needs to be diagnosed. He can also import image from gallery as well. The image is classified into healthy or diseased using SVM classifier. The application also provides detailed remedies to rectify the affected plant disease.

The system involves different phases for implementation purpose. The first phase is Dataset creation, in which both healthy and diseased plant leaf images are taken and perform background removal for each. Plant disease identification and classification [12] is done using image processing. Disease recognition is an important part of our system. Combined effect of Image processing and SVM classification can provide with the accurate solution. Dataset is divided into training set for learning the SVM classifier and test set to find the predictive accuracy of the system. Training set consists of $75 \%$ of the dataset and remaining $25 \%$ contribute to test set. It is independent of training test. The Second phase is image preprocessing, Image taken in a Smartphone is pre processed to extract its feature which is done with opencv image processing and Python 3 is used to build the code on opencv, which include conversion of image to grayscale, applying gaussian blurring, otsus thresholding for converting to binary image, morphological transformation to clean up otsus output, and then boundary extraction using contours. All these are performed to remove the noise for perfect classification. The Third phase includes feature extraction where features like area, perimeter, aspect ratio, rectangularity, entropy, contrast, etc. are extracted which is used to learn our training model. SVM library is used for the classification method. Initial stage of development, we focused only to four crops and their diseases this could be increased on the way. Tornado or django can be used to link our python code with android application. Figure 1 shows the proposed system methodology. Third Phase is Result and Remedy phase; we developed a library considering each crop and its disease. For diseases of a plant type the solution is provided within our library. The database is build from expertise knowledge and from study materials.

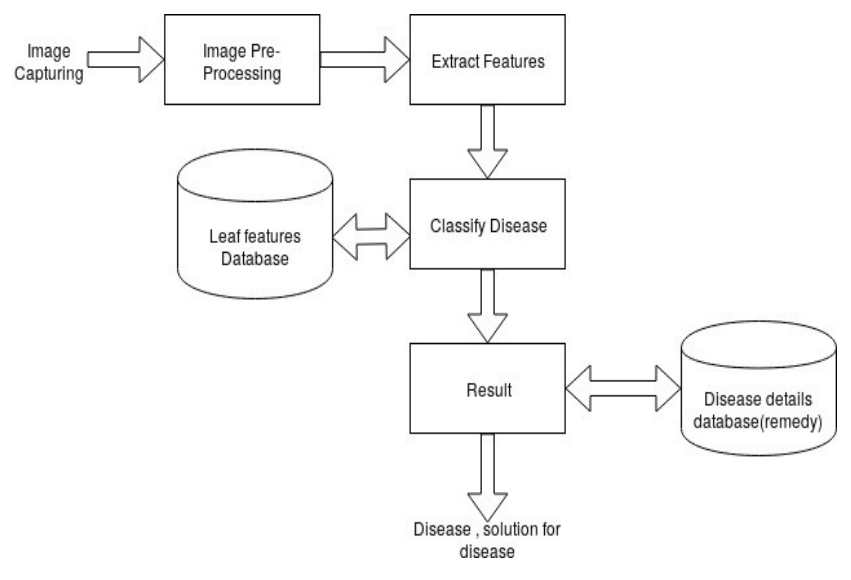

Figure 1: Proposed Methodology

The image can be captured either directly or can be imported from gallery. The image to be diagnosed is pre-processed to remove noise and extract features like area, perimeter, aspect ratio, etc. The extracted features of image are fed to the SVM classifier which is trained with our available dataset images. It then predicts whether the leaf is diseased or not.

Image taken in a Smartphone is pre processed to extract its feature which is done with opencv image processing and Python 3 is used to build the code on opencv, which include conversion of image to grayscale, applying gaussian blurring, otsus thresholding for converting to binary image, morphological transformation to clean up otsus ouput, and 
then boundary extraction using contours. All these are performed to remove the noise for perfect classification. The captured picture is transformed into grayscale and Gaussian blurring is used for smoothing the resultant image. Adaptive image thresholding is done using Otsu's thresholding method which outputs the binary image representing segmentation. Morphological Transformation is performed on the binary image to close the holes in it.

Morphological processing is performed to extract shape based, color based and texture based features for classification. Shape based features include calculating moments using contours, fitting in the best-fit rectangle and ellipse and then calculating features like Aspect ratio, rectangularity, circularity etc. Color based features include calculating color based features like mean, standard deviation of the RGB channels. Texture based features include using Haralick moments to calculate features such as contrast, correlation, entropy, etc. Feature extraction is shown in Figure 2.

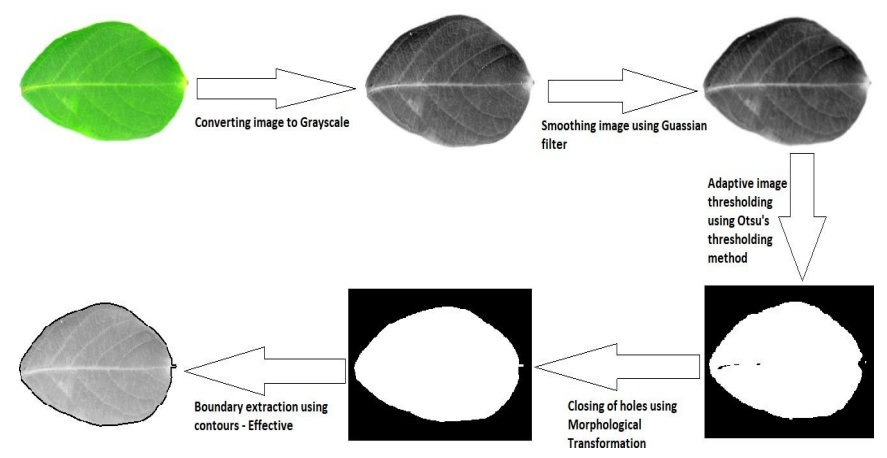

Figure 2: Feature Extraction

A classification model is constructed using the dataset collected. About $75 \%$ of images in the dataset are used as training set and remaining $25 \%$ used as test set. The classification algorithm develops the SVM classifier by analyzing or learning from the csv train set made up from feature extraction and their associated class labels. The class label indicates whether it is healthy or diseased. SVM can classify several data which can be either linear or nonlinear. It utilizes a nonlinear mapping to change [13] the first preparing information into a higher measurement. In this, it scans for the straight ideal isolating hyper plane. It finds the hyper plane utilizing fundamental preparing tuples called bolster vectors. In spite of the fact that the preparation time of even the quickest SVM can be very moderate, they are exceptionally precise with their capacity to display complex nonlinear choice limits. They are less prone to over fitting.

The constructed model is used to predict [14] class labels for given data, i.e., whether diseased or not. If diseased, it also provides remedies for the same. In this stage, the prescient precision of the classifier is assessed utilizing the test set which are autonomous of the preparation set utilized. The precision of the classifier is the degree of test tuples that are viably grouped. The related class name of the test tuple is contrasted and the classifier's forecast. Since the accuracy of the model is acceptable, it is used to classify future unseen data captured by the farmers.

Firstly when the mobile capture device is powered on, it becomes ready for capturing image. Thus the capture device, i.e. the mobile application, receives the captured image. Then we check two conditions, whether it has got an image or not. In the case when it has got an image, then that image is given to the processing system. The processing system is in charge for the background removal, preprocessing and feature extraction of the captured image. So in the first phase, it performs background removal, then saves that image and that image is given for preprocessing phase, whose output is given to feature extraction phase. Now the processing system is with features to be fed into SVM learning model (where SVM is already fed with features and classes of our training dataset). SVM model extracts knowledge and classifies according to the feature similarity. Then it predicts the class label of that unseen data. According to this class label, provide output to the mobile application that is when it is healthy, print out healthy or else when it is diseased, print the disease and remedy. Now when the device has not captured image, them reload the device.

\section{RESULTS AND DISCUSSIONS}

A plant leaf is healthy or diseased has been successfully identified and recommendations are provided for diseased. While we open our app, i.e. SasyaSneha which has the entry page consisting of choose image and diagnose buttons. When we click on choose image, we get many options to choose image. The options are directly capture from Camera or YouCam Perfect or import images from Gallery, Photos or from Drive. While clicking on any one option, we can take the photos as we wish to. The photo should be clear and focused with a white background. When the image is taken, we can crop the image so that the image can be focused. Next we have to click the Diagnose button for knowing whether the leaf is healthy or diseased. We have already trained the system with our datasets, so the system will not take much time because there is no training process going on each time press the Diagnose button. If the image is a diseased leaf, then it will show which the diseased plant is, what is the disease present in it and the remedies for curing its disease. Figure 3 and Figure 4 shows the happening with the front end of our application. 


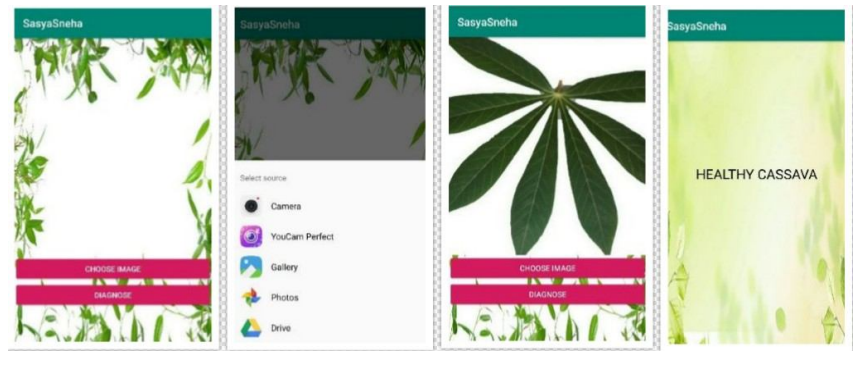

Figure 3: Result 1

In the back end, we are connecting our android studio code and our python programs using a web server. For this experiment we are using Tornado.

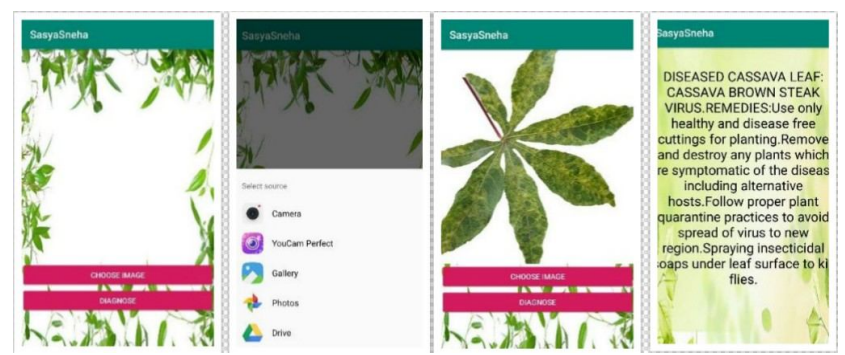

Figure 4: Result 2

\section{CONCLUSION}

The proposed method is implemented using an android application that captures the focused image of a leaf and predict whether it is healthy or diseased and predict its disease and remedies. SVM is learned with more than thousand images which comprises both healthy and diseased and thus classification of new input is done by SVM classifier from the learned knowledge. Predict the class label of unseen data and remedy for diseased. We have indicated how we extricate the pertinent highlights that speak to sickness from the leaf pictures and train SVM calculations to have the option to separate maladies dependent on these highlights. This proposed work thereby helps the farmers in early detection of diseases in more accurate manner without the help of an agronomist. Thus, this app stimulates the productivity of our country. In the presented work, the image processing only works when the image is took with a white background. So the image should be able to take irrespective of its background i.e. the image should be processed both in laboratory condition and also field condition. Here, the farmer itself has to focus the image and take the image while there is good clarity. It can be modified by automatically capturing the image while there is good focus and high clarity. Here we use English language as the language for communication. But the farmers may be illiterate and sometimes they can't understand English. So, the app can be modified by displaying the instruction in regional languages and translating to audio clips. Other thing is that, the app is focusing only on leaves of the plant. Only diseases in leaves are found. So, the app can be upgraded to find the diseases in any parts of plant and not only leaves. Now, we have a limitation that we have trained the system to identify only some of the specified diseases. So, the app can be modified to find all the disease of the plant.

\section{REFERENCES}

1. Mathai, Paul P., RV Siva Balan, and Ierin Babu. An efficient approach for item set mining using both utility and frequency based methods, International Journal of Applied Engineering Research 12.12 (2017): 3470-3473.

2. Mohanty, Sharada P., David P. Hughes, and Marcel Salathé. Using deep learning for image-based plant disease detection, Frontiers in plant science 7 (2016): 1419. https://doi.org/10.3389/fpls.2016.01419

3. S. Ravi Kumar, Vamsi Krishna Mangalapalli and Dr. Anurag. A survey on prediction approaches for epidemic disease outbreaks based on social media data, International Journal of Advanced Trends in Computer Science and Engineering, Volume 8, No.3, May-June 2019: 897-908 https://doi.org/10.30534/ijatcse/2019/86832019

4. Ch. Uma, M.Srinivasa C, Sri Ram C P, K.Babulu and D.O Prakash. Image Registration of Multi Model Enrollment using Mutual Information Technique, International Journal of Advanced Trends in Computer Science and Engineering, Vol. 7, No. 6, 2018:107-110 https://doi.org/10.30534/ijatcse/2018/07762018

5. Krizhevsky, Alex, Ilya Sutskever, and Geoffrey E. Hinton. Imagenet classification with deep convolutional neural networks, Advances in neural information processing systems. 2012

6. Johannes, Alexander, et al. Automatic plant disease diagnosis using mobile capture devices, applied on a wheat use case, Computers and electronics in agriculture 138 (2017): 200-209

https://doi.org/10.1016/j.compag.2017.04.013

7. Finlayson, Graham D., and Elisabetta Trezzi. Shades of gray and colour constancy, Color and Imaging Conference, Vol. 2004. No. 1. Society for Imaging Science and Technology, 2004

8. Xie, Xinhua, et al. A system for diagnosis of wheat leaf diseases based on Android smartphone, Optical Measurement Technology and Instrumentation, Vol. 10155. International Society for Optics and Photonics, 2016

https://doi.org/10.1117/12.2246919

9. Ferentinos, Konstantinos P. Deep learning models for plant disease detection and diagnosis, Computers and Electronics in Agriculture 145 (2018): 311-318 https://doi.org/10.1016/j.compag.2018.01.009

10. Lee, Sue Han, et al. Deep-plant: Plant identification with convolutional neural networks, 2015 IEEE 
International Conference on Image Processing (ICIP). IEEE, 2015

https://doi.org/10.1109/ICIP.2015.7350839

11. Nti, Isaac Kofi, Gyamfi Eric, and Yeboah Samuel Jonas. Detection of plant leaf disease employing image processing and gaussian smoothing approach, International Journal of Computer Applications 162.2 (2017): 20-25

https://doi.org/10.5120/ijca2017913260

12. Michael, Divya, Paul P. Mathai, and E. Abhidhat. Automatic vehicle detection and tracking in aerial surveillances using SVM, International Journal of Computer Applications 85.9 (2014)

https://doi.org/10.5120/14867-2990

13. Mathai, Shelmy, Paul P. Mathai, and K. A. Divya. Automatic 2D to 3D video and image conversion based on global depth map, 2015 IEEE International Conference on Computational Intelligence and Computing Research (ICCIC). IEEE, 2015 https://doi.org/10.1109/ICCIC.2015.7435781

14. Mathai, Paul P., RV Siva Balan, and Ierin Babu. An efficient approach for item set mining using both utility and frequency based methods, International Journal of Applied Engineering Research 12.12 (2017): 3470-3473 